\title{
Plk1 promotes the migration of human lung adenocarcinoma epithelial cells via STAT3 signaling
}

\author{
WEIJUAN YAN* ${ }^{*}$ HUIJIE YU* ${ }^{*}$ WEI LI, FENGSHENG LI, SINIAN WANG, NAN YU and QISHENG JIANG \\ Laboratory of Nuclear and Radiation Damage, The General Hospital of The Second \\ Artillery Corps of Chinese PLA, Beijing 100088, P.R. China
}

Received December 13, 2015; Accepted January 10, 2018

DOI: $10.3892 /$ ol.2018.9437

\begin{abstract}
Polo-like kinase (Plk)1 contributes to the development of human cancer via multiple mechanisms, such as promoting the migration of cancer cells. However, the mechanistic basis for the regulation of cell migration by Plk1 remains unknown. To address this question, the present study investigated the effect of Plk1 inhibition on the migration of human lung adenocarcinoma epithelial A549 cells and the molecular factors involved. A549 cells were treated with the Plk1 inhibitor, BI2536, and cell migration was evaluated with the wound-healing assay. The expression of matrix metallopeptidase (MMP)2, vascular endothelial growth factor (VEGF)A, total and phosphorylated signal transducer and activator of transcription (STAT) 3 was assessed by western blotting and reverse transcription-polymerase chain reaction following Plk1 knockdown and/or STAT3 overexpression. The interaction between Plk1 and STAT3 was evaluated by co-immunoprecipitation. The levels of MMP2 and VEGFA were decreased by treatment with Plk1 inhibitor. The phosphorylation of STAT3, which acts upstream of MMP2 and VEGFA, was also decreased by Plk1 knockdown, an effect that was abrogated by STAT3 overexpression. In addition, Plk1 was detected to bind with STAT3 either directly or as part of a complex by co-immunoprecipitation experiments. These results indicated that Plk1 may promote the migration of A549 cells via regulation of STAT3 signaling.
\end{abstract}

Correspondence to: Professor Qisheng Jiang or Dr Wei Li, Laboratory of Nuclear and Radiation Damage, The General Hospital of The Second Artillery Corps of Chinese PLA, 16 Xinjiekou Street, Xicheng, Beijing 100088, P.R. China

E-mail:wei_v@sina.com

E-mail: jqs598@sina.com

*Contributed equally

Key words: polo-like kinase 1, signal transducer and activator of transcription 3, migration, interaction, non-small cell lung cancer

\section{Introduction}

Lung cancer is a leading cause of cancer-associated mortality worldwide (1). Non-small cell lung carcinoma (NSCLC) accounts for $\sim 85 \%$ of lung cancer-associated mortalities worldwide (2,3). Despite recent improvements in chemo- and radiotherapy, the 5-year overall survival rate of patients with late-stage NSCLC is $<15 \%$ due to cancer recurrence and metastasis (4). Elucidating the molecular mechanisms of NSCLC progression may help to identify novel therapeutic targets, as well as novel treatment approaches.

The polo-like kinase (Plk) family in eukaryotes comprises highly conserved serine/threonine kinases (5). Plk1 controls mitotic entry, centrosome maturation, bipolar spindle formation and chromosome condensation and segregation (6). Plk1 has been linked to tumor aggressiveness and patient prognosis in numerous types of cancer, including NSCLC (7-9). Plk1 expression is upregulated in various cancer types (10-15), and this induces cell proliferation and malignant transformation (16). Previous studies have shown that Plk1 overexpression is involved in the invasion and migration of tumor cells in renal (17), colorectal (18) and bladder (19) cancer.

Signal transducer and activator of transcription (STAT) 3 of the Janus kinase-STAT signaling pathway is a latent transcription factor with a variety of functions (20). Constitutively-activated (phosphorylated) STAT3 protein contributes to tumor formation and metastasis in the majority of human cancer types $(21,22)$. Aberrant expression of Plk1 and STAT3 has been implicated in the migration of cancer cells (23-25). Plk1 functions in a transcriptional self-regulatory loop that involves the phosphorylation of forkhead box (Fox)M1 (26), while STAT3 and Plk1 exhibit cross-regulatory interactions in esophageal cancer (25). Based on these findings, it was speculated that STAT3 and Plk1 interact in a manner similar to Plk1 and FoxM1 to modulate the migration of tumor cells. To test this hypothesis, the present study investigated the effect of Plk1 inhibition on the migration of human lung adenocarcinoma epithelial A549 cells and the interaction between Plk1 and STAT3.

\section{Materials and methods}

Cell lines and culture conditions. A549 cells were isolated in the Laboratory of nuclear and radiation damage, The General Hospital of the Second Artillery Corps of Chinese 
PLA (Beijing, China), HeLa and 293T cells were donated by Professor Cheng Cao of the Academy of Military Medical Sciences (Beijing, China). All cells were cultured in Dulbecco's modified Eagle's medium (DMEM; Invitrogen; Thermo Fisher Scientific, Inc., Waltham, MA, USA) supplemented with $10 \%$ fetal bovine serum (Hyclone; GE Healthcare Life Sciences, Logan, UT, USA), $100 \mathrm{U} / \mathrm{ml}$ penicillin and $100 \mu \mathrm{g} / \mathrm{ml}$ streptomycin (Gibco; Thermo Fisher Scientific, Inc.). The cell cultures were incubated at $37^{\circ} \mathrm{C}$ in a humidified atmosphere of $5 \% \mathrm{CO}_{2}$.

Wound-healing assay. A549 cells ( $2 \times 10^{5}$ cells/well) were seeded in 6-well dishes at $80 \%$ confluence. After $16 \mathrm{~h}$, a scratch mark (wound) was made to the central area of confluent cells with a pipette tip. The cells were washed with serum-free DMEM, and the Plk1 inhibitor BI2536 $(0.1 \mu \mathrm{M})$ was added to the dishes. The cultures were incubated in serum-free medium at $37^{\circ} \mathrm{C}$ in a humidified atmosphere of $5 \% \mathrm{CO}_{2}$. The scratch area was captured under an inverted microscope (Olympus CX31; Olympus Corporation, Tokyo, Japan) and magnified by a computer-based microscopy imaging system (magnification, x200; Olympus Corporation) at 0, 24 and $48 \mathrm{~h}$ and the area of each wound was evaluated using Image-Pro Plus software (version 6.0, Media Cybernetics, Bethesda, MD, USA).

Small-interfering (si)RNA transfection. Plk1 and the control siRNA were purchased from GenePharma (GenePharma, Shanghai, China), and the sequences were 5'-AGATTGTGC CTAAGTCTCT-3' and 5'-UUCUCCGAACGUGUCACG UTT-3', respectively. The cells $\left(1 \times 10^{5}\right.$ cells/well) were seeded on a 6-well plate and transfected using Lipofectamine ${ }^{\circledR} 2000$ reagent (Invitrogen; Thermo Fisher Scientific, Inc.) following the manufacturer's protocols when $60 \%$ confluence was reached. The cells were harvested at 0,48 and $72 \mathrm{~h}$ following transfection. The cells were washed with cold phosphate buffered saline (PBS), centrifuged at $1,000 \times \mathrm{g}$ for $5 \mathrm{~min}$ at $4^{\circ} \mathrm{C}$, and then the supernatant was discarded, and the cell pellets were washed with PBS twice for reverse transcription-quantitative polymerase chain reaction (RT-qPCR) and western blot analysis. The experiments were repeated three times.

Total RNA isolation and RT-qPCR. Total RNA was isolated from cells using the RNeasy Mini kit (Qiagen GmbH, Hilden, German) according to the manufacturer's protocol, and then reverse-transcribed with random hexamers using the All-in-One First-Strand cDNA Synthesis kit (GeneCopoeia, Rockville, MD, USA). The expression of the transcripts was detected by RT-qPCR using the Real-time Premix EX TaqMan kit (Takara Biotechnology Co., Ltd., Dalian, China). All the experiments were carried out according to the manufacturers' protocols. The cycle threshold $(\mathrm{Cq})$ value for each target gene was normalized to the level of $\beta$-actin, and relative expression was calculated using the $2^{-\Delta \Delta \mathrm{Cq}}$ method (27). Each sample was analyzed in triplicate, and the experiment was repeated three times. The reaction conditions were as follows: $50^{\circ} \mathrm{C}$ for $2 \mathrm{~min}$ and $95^{\circ} \mathrm{C}$ for $5 \mathrm{~min}$, followed by 40 cycles of $95^{\circ} \mathrm{C}$ for $12 \mathrm{sec}$ and $60^{\circ} \mathrm{C}$ for $40 \mathrm{sec}$. The primer and probe sequences were as follows: Matrix metalloproteinase (MMP)2 forward, 5'-AAT CCATGATGGAGAGGCAGAC-3', reverse, 5'-CAGTCC GTCCTTACCGTCAAAG-3' and probe, 5'-CTTTGGCCG CTGGGGAGCATGG-3'; vascular endothelial growth factor
(VEGF)A forward, 5'-ACATCACCATGCAGATTATGC-3', reverse, 5'-GTCTTGCTCTATCTTTCTTTGGTC-3' and probe, 5'-CACCAAGGCCAGCACATAGGAGAGA-3'; and $\beta$-actin forward, 5'-TCTGGCAACGGTGAAGGTGACA-3', reverse, 5'-CACCTCCCCTGTGTGGACTT-3' and probe, 5'-AGCGAGCATCCCCCAAAGTTCACA-3'.

Co-immunoprecipitation (Co-IP). Cells were lysed in lysis buffer $(50 \mathrm{mM}$ Tris-HCl; $1 \mathrm{mM}$ phenylmethylsulfonyl fluoride; $1 \mathrm{mM}$ dithiothreitol; $10 \mathrm{mM}$ sodium fluoride; $10 \mu \mathrm{g} / \mathrm{ml}$ aprotinin; $10 \mu \mathrm{g} / \mathrm{ml}$ leupeptin; $10 \mu \mathrm{g} / \mathrm{ml}$ pepstatin $\mathrm{A}$; and $1 \%$ NonidetP-40). The cell lysates were kept on ice for $30 \mathrm{~min}$, and then centrifuged at $12,000 \mathrm{x}$ g for $15 \mathrm{~min}$ at $4^{\circ} \mathrm{C}$ and the soluble supernatants were collected.

For tagged Flag protein IP, cell supernatants were incubated with anti-Flag M2 beads (15 $\mu$; cat. no. A8592; Sigma-Aldrich; Merck KGaA, Darmstadt, Germany) for $4 \mathrm{~h}$ at $4^{\circ} \mathrm{C}$. Normal mouse IgG-Agarose (15 $\mu \mathrm{l}$; cat. no. A0910; Sigma-Aldrich; Merck KGaA) was used as the control and the condition were identical. Additionally, $15 \mu \mathrm{l}$ of the total supernatants $(5 \%, \mathrm{v} / \mathrm{v})$ was included as a control. Precipitates were then washed three times with lysis buffer and immunoblotted with horseradish peroxidase-conjugated anti-Flag (cat. no. A8592; 1:5,000 dilution; Sigma-Aldrich; Merck KGaA) or rabbit anti-STAT3 (cat. no. 4904; 1:1,000 dilution; Cell Signaling Technology, Inc., Danvers, MA, USA).

For STAT3 protein IP, cell supernatants were incubated with anti-STAT3 antibody ( $8 \mu \mathrm{l}$; cat. no. 4904; Cell Signaling Technology, Inc.) for $2 \mathrm{~h}$ at $4^{\circ} \mathrm{C}$, and then incubated with Protein A/G agarose (20 $\mu \mathrm{l}$; cat. no. 2336; Santa Cruz Biotechnology, Inc., Dallas, TX, USA) for $12 \mathrm{~h}$ at $4^{\circ} \mathrm{C}$. The normal rabbit IgG (cat. no. A2909, $4 \mu$ l; rabbit; Santa Cruz Biotechnology, Inc.) was used as the control and the conditions were identical. Additionally, $15 \mu 1$ of the total supernatants $(5 \%, v / v)$ was included as a control. Precipitates were then washed three times with lysis buffer and immunoblotted with the indicated antibodies (anti-Plk1 and anti-STAT3) for western blot analysis.

Western blot analysis. To isolate the protein, the cells were first washed twice in PBS at $48 \mathrm{~h}$ after transferation and lysed in RIPA lysis buffer (ProMab Biotechnologies, Inc., Richmond, CA, USA; 50 mM Tris-HCI pH 7.6, $150 \mathrm{mM}$ sodium chloride, $1.0 \%$ NP-40, $1 \%$ sodium deoxychlorate, and $0.1 \%$ SDS) plus protease inhibitors. The lysates were kept on ice for $30 \mathrm{~min}$, and then centrifuged at $14,000 \mathrm{x} \mathrm{g}$ for $15 \mathrm{~min}$. The surpernatant was collected and then $20 \mu \mathrm{g}$ of each of the proteins was separated by SDS-PAGE on $10 \%$ gels and transferred onto nitrocellulose membranes The proteins were electrotransferred to polyvinylidene difluoride membranes, blocked in 5\% non-fat milk (Bio-Rad Laboratories, Inc., Hercules, CA, USA) at room temperature for $1 \mathrm{~h}$ and incubated with primary antibodies overnight at $4^{\circ} \mathrm{C}$. Primary antibodies against the following targets were used: Anti-Plk1 (cat. no. J3108, mouse; 1:1,000; Santa Cruz Biotechnology, Inc.); anti-STAT3 and anti-phosphorylated (p-)STAT3 Y705 (cat. no. 9145, rabbit; 1:1,000; Cell Signaling Technology, Inc.); horseradish peroxidase (HRP)-conjugated anti- $\beta$-actin (cat. no. A3584, 1:10,000; Sigma-Aldrich; Merck KGaA); anti-MMP2 (cat. no. BS1236, rabbit; 1:500; Bioworld 

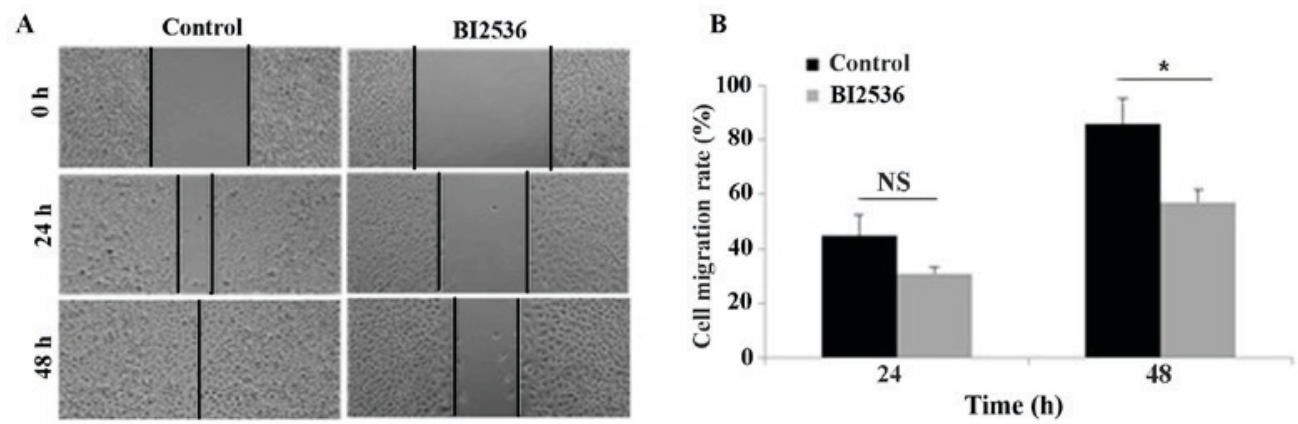

Figure 1. Inhibition of Polo-like kinase 1 suppresses the migration of A549 cells. The migration of BI2536 (0.1 $\mu \mathrm{M})$-treated and untreated A549 cells was evaluated at 0,24 and $48 \mathrm{~h}$ using wound-healing assay. (A) Representative images of migrating cells (magnification, x10). (B) Quantitative analysis of cell migration rates. ${ }^{*} \mathrm{P}<0.05$ vs. the control group (Student's t-test).

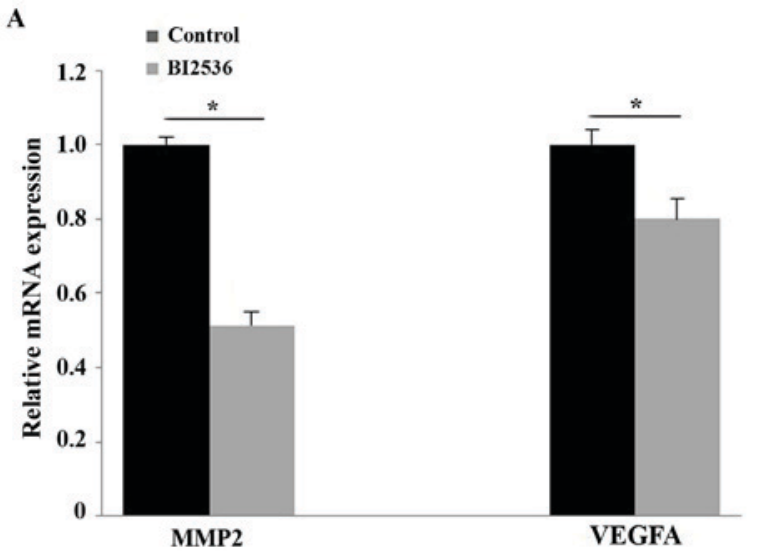

B
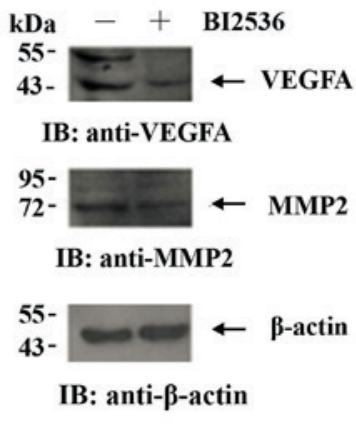

Figure 2. Inhibition of Polo-like kinase 1 suppresses the expression of MMP2 and VEGFA. The levels of MMP2 and VEGFA (A) transcripts and (B) protein expression in BI2536-treated A549 cells were analyzed at $12 \mathrm{~h}$ by reverse transcription-quantitative polymerase chain reaction and western blotting, respectively. "P<0.05 vs. the control group (Student's t-test). MMP2, matrix metalloproteinase 2; VEGFA, vascular endothelial growth factor A.

Technology, Inc., St. Louis Park, MN, USA) and anti-VEGFA (cat. no. BS2431, rabbit; 1:500; Bioworld Technology, Inc.). Subsequently, the membranes were washed four times for 5 min each with PBS containing $0.05 \%$ Tween-20 and incubated with anti rabbit or anti mouse horseradish peroxidase conjugated secondary antibodies (HRP-conjugated anti-mouse and HRP-conjugated rabbit antibodies (GE Healthcare; dilution, 1:2,000) for $1 \mathrm{~h}$ at room temperature. Finally, the protein bands were visualized using an enhanced chemiluminescence detection reagent (GE Healthcare, Little Chalfont, UK) and detected using a ChemiDoc imager (Bio-Rad Laboratories, Inc.).

Double luciferase reporter assay. HEK $293 \mathrm{~T}$ cells $\left(5 \times 10^{4}\right.$ cells/well) were seeded on 24 -well plates and transfected with STAT3-flag $(0.15 \mu \mathrm{g})$ and Plk1-flag $(0,0.15$ and $0.25 \mu \mathrm{g}$ ) using Lipofectamine ${ }^{\circledR} 2000$ reagent (Invitrogen; Thermo Fisher Scientific, Inc.), along with a luciferase reporter gene $(0.15 \mu \mathrm{g})$ and pRL-TK plasmid $(0.0003 \mu \mathrm{g})$. The relative firefly luciferase activity was normalized to Renilla luciferase activity. Following incubation for $24 \mathrm{~h}$, the cells were lysed and the luciferase activity was analyzed by the dual luciferase assay kit (Promega Corporation, Madison, WI, USA) according to the manufacturer's protocol. The data are presented as the mean \pm standard deviation (SD) of three independent experiments performed in triplicate.
Statistical analysis. Statistical analyses were performed using SPSS software (version 19.0; IBM SPSS, Armonk, NY, USA). The data are presented as the mean $\pm \mathrm{SD}$. A two-tailed Student's t-test for paired data was used to compare the means of two groups. $\mathrm{P}<0.05$ was considered to indicate a statistically significant difference.

\section{Results}

Inhibition of Plk1 suppresses the migration of A549 cells. The effect of Plk1 inhibition on the migration of A549 cells was evaluated with the wound-healing assay (Fig. 1A). Treatment with BI2536, which is an inhibitor of Plk1, for $48 \mathrm{~h}$ decreased migration in control cells from $85.60 \pm 9.50$ to $57.10 \pm 4.48 \%$, indicating that the inhibition of Plk1 is able to block the migration of human lung adenocarcinoma epithelial cancer cells (Fig. 1B).

Inhibition of Plk1 suppresses MMP2 and VEGFA expression. MMP2 and VEGFA have been implicated in the invasion and migration of tumor cells (28-30). The effect of Plk1 inhibition on the mRNA and protein expression of these factors in BI2536-treated cells were analyzed with RT-qPCR and western blotting, respectively. The expression of MMP2 and VEGFA was decreased in the cells that were treated with BI2536 compared with control cells (Fig. 2), providing additional evidence that Plk1 modulates the migration of tumor cells. 
A

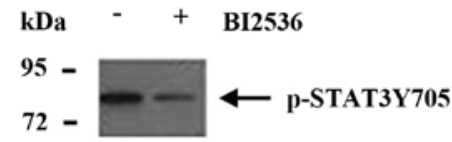

IB: anti-p-STAT3Y705

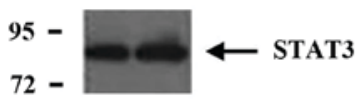

IB: anti-STAT3

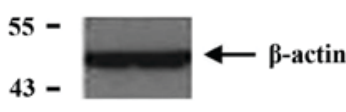

IB: anti- $\beta$-actin
B

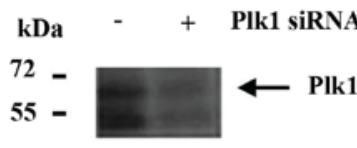

IB: anti-PIk1
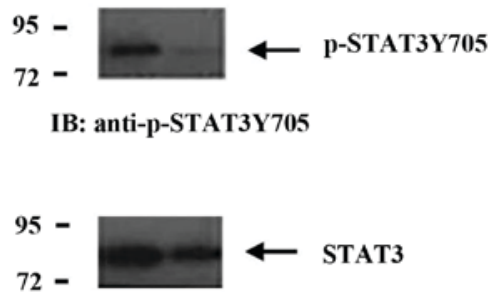

IB: anti-STAT3

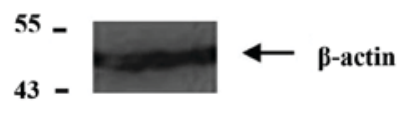

IB: anti- $\beta$-actin

Figure 3. Pharmacological inhibition or knockdown of Plk1 blocks the phosphorylation of STAT3. (A) A549 cells were treated with BI2536 (0.1 $\mu \mathrm{M})$ or left untreated for $12 \mathrm{~h}$, and subsequently the lysates were analyzed by western blotting. (B) A549 cells were transfected twice at a 12-h interval with a small-interfering RNA that targets Plk1. After 48 h, the lysates were probed by western blotting. p, phosphorylated; Plk1, polo-like kinase 1; STAT3, signal transducer and activator of transcription 3 .

Suppression of Plk1 blocks the phosphorylation of STAT3. MMP2 and VEGFA are downstream target genes of STAT3 $(31,32)$. Therefore, the effect of Plk1 inhibition on STAT3 and p-STAT3 expression was investigated. Treatment with BI2536 decreased the level of p-STAT3 without altering the level of STAT3 (Fig. 3A). siRNA-mediated knockdown of Plk1 resulted in the downregulation of STAT3 and p-STAT3 expression (Fig. 3B) and activity, as determined by the luciferase assay. This was confirmed by overexpressing STAT3 in BI2536-treated A549 cells, which reversed the downregulation of p-STAT3, VEGFA and MMP2 mediated by Plk1 inhibition compared with the cells that were transfected with the control plasmid (Fig. 4). These results suggested that Plk1 modulates the migration of tumor cells via STAT3 signaling.

Plk1 interacts with STAT3. The inhibition of Plk1 blocked the phosphorylation of STAT3, while the level of STAT3 was unchanged, suggesting a possible interaction between the two proteins. To investigate this possibility, the interaction between Plk1 and STAT3 was examined by co-IP in HeLa cells, which exhibit higher endogenous expression of Plk1 compared with A549 cells. HeLa cell lysates immunoprecipitated with anti-STAT3 antibody were analyzed by western blotting using an anti-Plk1 antibody. Plk1 was detected in the immunoprecipitate obtained using anti-STAT3 antibody, but not that obtained using rabbit IgG (Fig. 5A). To confirm the interaction between STAT3 and Plk1, 293T cells were transfected with plasmids expressing flag-Plk1 or flag-vector, and cell lysates precipitated with anti-flag antibody were probed with anti-STAT3 antibody. The western blot analysis revealed that STAT3 is able to interact with Plk1 (Fig. 5B). In addition, the co-transfection of Plk1 and STAT3-luciferase reporter plasmids increased the relative luciferase activity compared

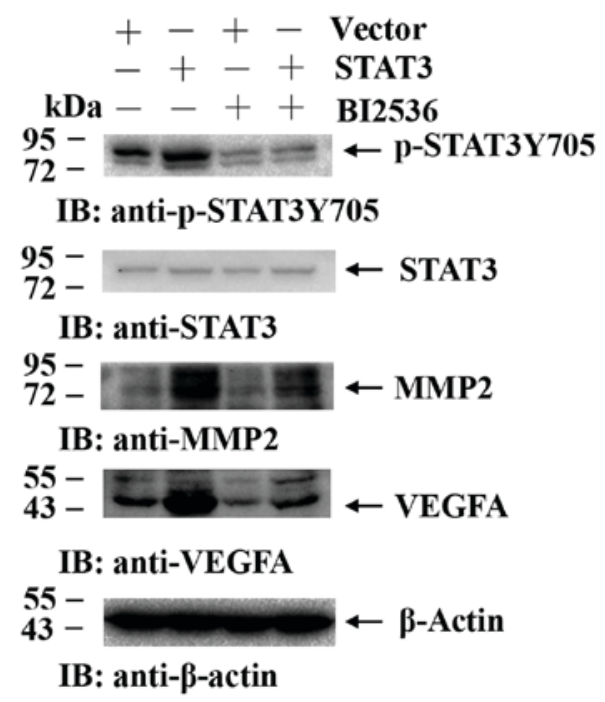

Figure 4. The effect of polo-like kinase 1-mediated inhibition on the migration of A549 cells as STAT3 overexpression. BI2536-treated $(0.1 \mu \mathrm{M})$ or untreated cells were transfected with a STAT3-expressing plasmid. The lysates were analyzed by western blotting. IB, immunoblotting; p-, phosphorylated; STAT3, signal transducer and activator of transcription; MMP2, matrix metalloproteinase 2; VEGFA, vascular endothelial growth factor A.

with the control vectors (Fig. 5C). These results suggested that Plk1 enhances STAT3 activity in tumor cells.

\section{Discussion}

Cancer metastasis is complex and regulated by multiple factors (33-35). Plk1 has been shown to promote invasion and migration in several types of cancer $(13,14)$, For example, 
A

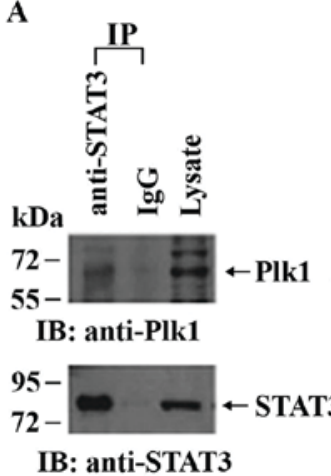

B

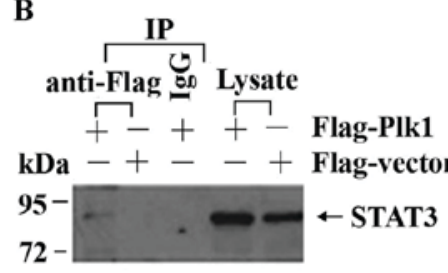

IB: anti-STAT3

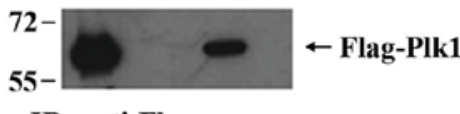

C

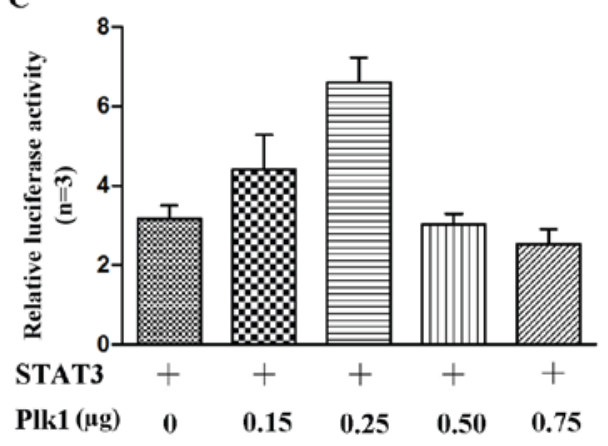

Figure 5. Plk1 interacts with STAT3. (A) HeLa cell lysates were immunoprecipitated with anti-STAT3 or anti-flag antibodies and analyzed by western blotting with indicated antibodies. Normal rabbit IgG was used as the control (B). 293T cells were transfected with flag-Plk1 or flag-vector plasmids. Anti-flag immunoprecipitates were analyzed by western blotting with anti-STAT3 or anti-flag antibodies. (C) 293T cells were transfected with STAT3 luciferase reporter plasmids, along with flag-Plk1 plasmid. Relative firefly luciferase activity was normalized to Renilla luciferase activity. After 24 h, the relative luciferase activity was analyzed. Plk1, polo-like kinase 1; STAT3, signal transducer and activator of transcription 3; IP, immunoprecipitate; Ig, immunoglobulin.

Han et al (18) reported that Polo-like kinase 1 was overexpressed in colorectal cancer and participated in the invasion and migration of colorectal cancer cells. However, the underlying mechanism is unclear. The present study indicates that the inhibition of Plk1 suppressed the migration of A549 cells and decreased the expression of MMP2 and VEGFA and phosphorylation of STAT3. Furthermore, to the best of our knowledge, the present study presents for the first time the interaction of Plk1 and STAT3 to regulate cell migration.

The overexpression of Plk1 is associated with a low survival rate and poor prognosis in various types of cancer $(16,17,19)$, which can be attributed to its role in promoting the invasion and migration of cancer cells (17-19). Consistent with these findings, in the present study, it was observed that the migration of A549 cells was blocked by treatment with BI2536, which is a small-molecular inhibitor of Plk1.

MMP2 belongs to a family of structurally-related enzymes that degrade the components of the extracellular matrix during tumor invasion and metastasis (36). The overexpression of MMP2 is observed in solid tumors of different origins, including metastatic lesions (37-40), this is positively associated with tumor size, depth of invasion, lymphatic and venous invasion and lymph node metastasis in gastric carcinoma (41). In the present study, MMP2 expression was downregulated upon the inhibition of Plk1. VEGFA is a potent inducer of angiogenesis, a process that allows solid tumors to acquire nutrients for continuous growth and metastasis (42). VEGFA has been shown to be upregulated in the majority of malignancies and promotes the progression and metastasis of various types of cancer (43). Conversely, the knockdown of VEGFA suppressed lymph node metastasis in a murine metastatic mammary cancer model (44). In the present study, the level of VEGFA was observed to be markedly reduced by Plk1 inhibition. These results indicated that the inhibition of Plk1 may suppress the migration of A549 cells by negatively regulating the expression of MMP2 and VEGFA.

The activation of STAT3 leads to the upregulation of MMP2 and VEGFA, thereby enhancing the migration and invasion of tumor cells (36). STAT3 is constitutively activated in numerous types of human cancer, usually by phosphorylation of a conserved tyrosine residue in response to extracellular signals from cytokines and growth factors (45). Activated p-STAT3 forms homo- or heterodimers that translocate into the nucleus and induce the transcription of target genes, including MMP2 and VEGFA. The constitutive activation of STAT3 has also been shown to modulate cancer invasion and metastasis (46-48), which is an effect that is mitigated by the inhibition of STAT3 signaling. Therefore, it was hypothesized that the inhibition of Plk1 suppresses MMP2 and VEGFA by negatively regulating STAT3 expression or activation. This was supported by the observations that the levels of phosphorylated STAT3 but not total protein expression, decreased upon pharmacological inhibition of Plk1, and that the overexpression of STAT3 countered the effects of Plk1 inhibition in A549 cells. The present results are consistent with those of a previous study (26) on esophageal cancer cells, which reported that Plk1 knockdown or pharmacological inhibition suppressed STAT3 activity. However, one difference between this earlier study and the present study is that BI2536 treatment reduced STAT3 expression in esophageal cancer cells, whereas no change in STAT3 level was observed in A549 cells. This may be attributable to intrinsic differences between the two cell types.

A previous study (49) reported an association between Plk1 and regulation of the G2/M transcriptional network in mammalian cells. Plk1 has been shown to be involved in a positive-feedback loop with its binding partner FoxM1, a transcription factor that controls the expression of various M-phase genes, including Plk1. The Plk1-FoxM1 complex allows the direct phosphorylation of FoxM1 by Plk1 at the G2/M phase. The activation of FoxM1 is required for Plk1 expression, which is enhanced by FoxM1. STAT3 was reported to directly activate Plk1 transcription in esophageal cancer cells $(25,50)$. Therefore, it was speculated that Plk1 and STAT3 interact in a manner similar to Plk1 and FoxM1. Indeed, binding between Plk1 and STAT3 was observed between Plk1 and STAT3 in co-IP experiments in the present study. Furthermore, the luciferase reporter assay revealed that Plk1 enhanced the activity of STAT3. However, Plk1 is a Ser/Thr kinase, and it promotes tyrosine phosphorylation of STAT3 $(51,52)$, therefore the interaction between Plk1 and STAT3 may be indirect. Additional experiments such as protein microarrays or cross-linking are 
required to determine whether the interaction between the two proteins is direct or indirect.

The results of the present study demonstrated that Plk1 inhibition suppresses the migration of A549 cell via the downregulation of STAT3 signaling and the target genes MMP2 and VEGFA. These findings suggested that the inhibition of Plk1 may prevent the migration and metastasis of cancer cells and can therefore be an effective therapeutic approach for the treatment of NSCLC.

\section{Acknowledgements}

The authors thank Professor Cheng Cao from the Institute of Biotechnology of Academy of Military Medical Sciences for providing antibodies against Plk1 and $\beta$-actin, and Dr Quanbin Xu from the Institute of Biotechnology of Academy of Military Medical Sciences and Professor Ling Gao from Key Laboratory of Radiological Protection and Nuclear Emergency, National Institute for Radiological Protection, China Centers for Disease Control for providing the Plk1 inhibitor BI2536, A549 cells and the overexpression vector.

\section{Funding}

The present study was supported by National Nature Science Foundation of China (grant nos. 81602796 and 31770914) and Major Technology Project of Military Logistics (grant no. AEP17J001).

\section{Availability of data and materials}

All data generated or analysed during this study are included in this published article.

\section{Authors' contributions}

WL and QJ designed the experiments and checked the manuscript. WY performed the experiments, prepared the data and wrote and edited the manuscript. HY performed the experiments and programmed the software. FL designed and performed the experiments. SW and NY performed the experiments.

\section{Ethics approval and consent to participate}

Not applicable.

\section{Patient consent for publication}

Not applicable.

\section{Competing interests}

The authors declare that they have no competing interests.

\section{References}

1. Kanwal M, Ding XJ and Cao Y: Familial risk for lung cancer. Oncol Lett 13: 535-542, 2017.

2. Torre LA, Bray F, Siegel RL, Ferlay J, Lortet-Tieulent J and Jemal A: Global cancer statistics, 2012. CA Cancer J Clin 65: 87-108, 2015.
3. Stinchcombe TE and Socinski MA: Current treatments for advanced stage non-small cell lung cancer. Proc Am Thorac Soc 6: 233-241, 2009.

4. Hu Z, Chen X, Zhao Y, Tian T, Jin G, Shu Y, Chen Y, Xu L, Zen K, Zhang C and Shen H: Serum microRNA signatures identified in a genome-wide serum microRNA expression profiling predict survival of non-small-cell lung cancer. J Clin Oncol. 28: $1721-1726,2010$

5. Combes G, Alharbi I, Braga LG and Elowe S: Playing polo during mitosis: PLK1 takes the lead. Oncogene 34: 4819-4827, 2017.

6. Song B, Liu XS and Liu X: Polo-like kinase 1 (Plk1): An unexpected player in DNA replication. Cell Div 7: 3, 2012.

7. Liu Z, Sun Q and Wang X: PLK1, a potential target for cancer therapy. Transl Oncol 10: 22-32, 2017.

8. Xu C, Li S, Chen T, Hu H, Ding C, Xu Z, Chen J, Liu Z, Lei Z, Zhang HT, et al: miR-296-5p suppresses cell viability by directly targeting PLK1 in non-small cell lung cancer. Oncol Rep 35: 497-503, 2016.

9. Wang XH, Lu Y, Liang JJ, Cao JX, Jin YQ, An GS, Ni JH, Jia HT and Li SY: MiR-509-3-5p causes aberrant mitosis and anti-proliferative effect by suppression of PLK1 in human lung cancer A549 cells. Biochem Biophys Res Commun 478: 676-682, 2016.

10. Wang H, Tian C, Xu Y, Xie WL, Zhang J, Zhang BY, Ren K, Wang K, Chen C, Wang SB, et al: Abortive cell cycle events in the brains of scrapie-infected hamsters with remarkable decreases of PLK3/Cdc25C and increases of PLK1/cyclin B1. Mol Neurobiol 48: 655-668, 2013.

11. King SI, Purdie CA, Bray SE, Quinlan PR, Jordan LB, Thompson AM and Meek DW: Immunohistochemical detection of Polo-like kinase-1 (PLK1) in primary breast cancer is associated with TP53 mutation and poor clinical outcom. Breast Cancer Res 14: R40, 2012.

12. Takahashi T, Sano B, Nagata T, Kato H, Sugiyama Y, Kunieda K, Kimura M, Okano Y and Saji S: Polo-like kinase 1 (PLK1) is overexpressed in primary colorectal cancers. Cancer Sci 94: 148-152, 2003.

13. Medema RH, Lin CC and Yang JC: Polo-like kinase 1 inhibitors and their potential role in anticancer therapy, with a focus on NSCLC. Clin Cancer Res 17: 6459-6466, 2011.

14. Luo J and Liu X: Polo-like kinase 1, on the rise from cell cycle regulation to prostate cancer development. Protein Cell 3: 182-197, 2012.

15. Sun W, Su Q, Cao X, Shang B, Chen A, Yin H and Liu B: High expression of polo-like kinase 1 is associated with early development of hepatocellular carcinoma. Int J Genomics 2014: 312130, 2014.

16. Ito $\mathrm{Y}$, Yoshida $\mathrm{H}$, Matsuzuka F, Matsuura N, Nakamura $\mathrm{Y}$, Nakamine H, Kakudo K, Kuma K and Miyauchi A: Polo-like kinase 1 (PLK1) expression is associated with cell proliferative activity and cdc2 expression in malignant lymphoma of the thyroid. Anticancer Res 24: 259-263, 2004.

17. Zhang G, Zhang Z and Liu Z: Polo-like kinase 1 is overexpressed in renal cancer and participates in the proliferation and invasion of renal cancer cells. Tumour Biol 34: 1887-1894, 2013.

18. Han DP, Zhu QL, Cui JT, Wang PX, Qu S, Cao QF, Zong YP, Feng B, Zheng MH and Lu AG: Polo-like kinase 1 is overexpressed in colorectal cancer and participates in the migration and invasion of colorectal cancer cells. Med Sci Monit 18: BR237-246, 2012.

19. Zhang Z, Zhang G and Kong C: High expression of polo-like kinase 1 is associated with the metastasis and recurrence in urothelial carcinoma of bladder. Urol Oncol 31: 1222-1230, 2013.

20. Zhao M, Gao FH, Wang JY, Liu F, Yuan HH, Zhang WY and Jiang B: JAK2/STAT3 signaling pathway activation mediates tumor angiogenesis by upregulation of VEGF and bFGF in non-small-cell lung cancer. Lung Cancer 73: 366-374, 2011.

21. Yu Y, Zhao Q, Wang Z and Liu XY: Activated STAT3 correlates with prognosis of non-small cell lung cancer and indicates new anticancer strategies. Cancer Chemother Pharmacol 75: 917-922, 2015.

22. Liu RY, Zeng Y, Lei Z, Wang L, Yang H, Liu Z, Zhao J and Zhang HT: JAK/STAT3 signaling is required for TGF-beta-induced epithelial-mesenchymal transition in lung cancer cells. Int J Oncol 44: 1643-1651, 2014.

23. Morry J, Ngamcherdtrakul W, Gu S, Reda M, Castro DJ, Sangvanich T, Gray JW and Yantasee W: Targeted treatment of metastatic breast cancer by PLK1 siRNA delivered by an antioxidant nanoparticle platform. Mol Cancer Ther 4: 763-772, 2017. 
24. Xu L, Zhou R, Yuan L, Wang S, Li X, Ma H, Zhou M, Pan C, Zhang $J$ and Huang N: IGF1/IGF1R/STAT3 signaling-inducible IFITM2 promotes gastric cancer growth and metastasis. Cancer Lett 393: 76-85, 2017.

25. Zhang Y: Reciprocal Activation between PLK1 and Stat3 contributes to survival and proliferation of esophageal cancer cells. Gastroenterology 142: 521, 2012.

26. Pomerening JR: Positive-feedback loops in cell cycle progression. FEBS Lett 583: 3388-3396, 2009.

27. Livak KJ and Schmittgen TD: Analysis of relative gene expression data using real-time quantitative PCR and the 2 (-Delta Delta C(T)) method. Methods 25: 402-408, 2001.

28. Fossey SL, Bear MD, Kisseberth WC, Pennell M and London CA Oncostatin M promotes STAT3 activation, VEGF production, and invasion in osteosarcoma cell lines. BMC Cancer 11: 125, 2011.

29. Yang L, Song X, Zhu J, Li M, Ji Y, Wu F, Chen Y, Cui X, Hu J, Wang L, et al: Tumor suppressor microRNA-34a inhibits cell migration and invasion by targeting MMP-2/MMP-9/FNDC3B in esophageal squamous cell carcinoma. Int J Oncol 51: 378, 2017.

30. Gong J, Zhu S, Zhang Y and Wang J: Interplay of VEGFa and MMP2 regulates invasion of glioblastoma. Tumor Biol 35: 11879-11885, 2014.

31. Kesanakurti D, Chetty C, Dinh DH, Gujrati M and Rao JS: Role of MMP-2 in the regulation of IL-6/Stat3 survival signaling via interaction with $\alpha 5 \beta 1$ integrin in glioma. Oncogene 32: 327-340, 2013.

32. Shen X, Tang J, Hu J, Guo L, Xing Y and Xi T: MiR-424 regulates monocytic differentiation of human leukemia U937 cells by directly targeting CDX2. Biotechnol Lett 35: 1799-1806, 2013.

33. Hsu F, Caluwe AD, Anderson D, Nichol A, Toriumi T and Ho C: Patterns of spread and prognostic implications of lung cancer metastasis in an era of driver mutations. Curr Oncol 24: 228-233, 2017.

34. Su SC, Hsieh MJ, Yang WE, Chung WH, Reiter RJ and Yang SF: Cancer metastasis: Mechanisms of inhibition by melatonin. J Pineal Res 62: e12370, 2017.

35. BJ Scott, Oberheimbush NA and Kesari S: Leptomeningeal metastasis in breast cancer-a systematic review. Oncotarget 4: 3740-3747, 2016.

36. Roomi MW, Monterrey JC, Kalinovsky T, Rath M and Niedzwiecki A: Inhibition of invasion and MMPs by a nutrient mixture in human cancer cell lines: A correlation study. Exp Oncol 32: 243-248, 2010.

37. Groblewska M, Mroczko B, Gryko M, Kedra B and Szmitkowski M: Matrix metalloproteinase 2 and tissue inhibitor of matrix metalloproteinases 2 in the diagnosis of colorectal adenoma and cancer patients. Folia Histochem Cytobiol 48 : 564-571, 2010.

38. Duan W, Li R, Ma J, Lei J, Xu Q, Jiang Z, Nan L, Li X, Wang Z, Huo $X$, et al: Overexpression of Nodal induces a metastatic phenotype in pancreatic cancer cells via the Smad2/3 pathway. Oncotarget 6: 1490-1506, 2015.
39. Zhang Y, Pan T, Zhong X and Cheng C: Androgen receptor promotes esophageal cancer cell migration and proliferation via matrix metalloproteinase 2. Tumour Biol 36: 5859-5864, 2015.

40. Zheng H, Takahashi H, Murai Y, Cui Z, Nomoto K, Niwa H, Tsuneyama K and Takano Y: Expressions of MMP-2, MMP-9 and VEGF are closely linked to growth, invasion, metastasis and angiogenesis of gastric carcinoma. Anticancer Res 26: 3579-3583, 2006

41. Zhu Y, Yu X, Fu H, Wang P, Zheng X and Wang Y: MicroRNA-21 is involved in ionizing radiation-promoted liver carcinogenesis. Int J Clin Exp Med 3: 211, 2010.

42. Ferrara N, Gerber HP and LeCouter J: The biology of VEGF and its receptors. Nature Med 9: 669-676, 2003.

43. Shibata MA, Morimoto J, Shibata E and Otsuki Y: Combination therapy with short interfering RNA vectors against VEGF-C and VEGF-A suppresses lymph node and lung metastasis in a mouse immunocompetent mammary cancer model. Cancer Gene Ther 15: 776-786, 2008

44. Miao JW, Liu LJ and Huang J: Interleukin-6-induced epithelial-mesenchymal transition through signal transducer and activator of transcription 3 in human cervical carcinoma. Int J Oncol 45: 165-176, 2014

45. Yan LI,Li LI, LiQ,DI W, Shen W, Zhang L and Guo H: Expression of signal transducer and activator of transcription 3 and its phosphorylated form is significantly upregulated in patients with papillary thyroid cancer. Exp Ther Med 9: 2195-2201, 2015.

46. Li C, Wang Z, Liu Y, Wang P and Zhang R: STAT3 expression correlates with prognosis of thymic epithelial tumors. J Cardiothoracic Surg 8: 92, 2013.

47. Park JK, Hong R, Kim KJ, Lee TB and Lim SC: Significance of p-STAT3 expression in human colorectal adenocarcinoma. Oncol Rep 20: 597-604, 2008.

48. Zhang M, Zhu GY, Gao HY, Zhao SP and Xue Y: Expression of tissue levels of matrix metalloproteinases and tissue inhibitors of metalloproteinases in gastric adenocarcinoma. J Surg Oncol 103: 243-247, 2011.

49. Zheng F, Malureanu L, Huang J, Wang W, Li H, van Deursen JM, Tindall DJ and Chen J: Plk1-dependent phosphorylation of FoxM1 regulates a transcriptional programme required for mitotic progression. Nat Cell Biol 9:1076-1082, 2008.

50. Dibb M, Han N, Choudhury J, Hayes S, Valentine H, West C, Ang YS and Sharrocks AD: The FOXM1-PLK1 axis is commonly upregulated in oesophageal adenocarcinoma. Brit J Cancer 107: 1766-1775, 2012.

51. Guo K, Yin G, Zi X-H and Yan W-G: A study on expression and Tyrosine 705 phosphorylation of STAT3 in focal cerebral ischemia-reperfusion rat model and its role in neuronal apoptosis. Trop J Pharma Res 2: 267, 2016. doi: 10.4314/tjpr.v15i2.7.

52. Shin SB, Woo SU, Chin YW, Jang YJ and Yim H: Sensitivity of TP53-Mutated cancer cells to the Phytoestrogen Genistein is associated with direct inhibition of Plk1 activity. J Cell Physiol 232: 2818-2828, 2017 